\title{
Long Term Evolution (LTE) Scheduling Algorithms in Wireless Sensor Networks (WSN)
}

\author{
A.I.A. Jabbar, PhD \\ Engineering College \\ Department of Electrical Engineering \\ Mosul University
}

\author{
Fawaz Y. Abdullah \\ Engineering College \\ Department of Electrical Engineering \\ Mosul University
}

\begin{abstract}
Many applications of wireless sensor network such as smart metering, environment monitoring and health care [1] use a large number of sensor nodes, which generate a huge amount of traffic with the specification of the different QoS requirements. Broadband transmission can be achieved through the mobile systems. Long Term Evolution (LTE) is the main efficient broadband technology in current mobile communications. Introducing Wireless sensor networks into the Long Term Evolution (LTE) networks, can be achieved successfully but with some challenges, such as the traffic overload due to a large number of sensor nodes. Traffic scheduling plays an important role in LTE technology; this can be achieved by assigning the shared resources among users in an efficient and optimized manner. This paper discusses the effect of many types of scheduling algorithms on the downlink performance, this can be done in terms of throughput, block error rate (BLER) and fairness measurements using a MATLAB-based system level Simulator is provide which from Vienna University under License Agreement for "Academic Usage" [8]. An effective radio scheduler to optimize the distribution of radio resources among the sensor nodes is also studied. The guaranteed QoS user demands can be considered as a great challenge in the current field of research.
\end{abstract}

\section{General Terms}

Computer Network, Wireless Networks, Modeling, Scheduling, Algorithms.

\section{Keywords}

LTE, WSN, scheduling algorithms.

\section{INTRODUCTION}

Wireless sensors can be connected directly (through a gateway) to an eNodB (LTE base station) or via unstructured networking, ad-hoc networking or cognitive radio techniques $[1,2]$. In this paper, sensor nodes (SNs) are placed in locations where LTE, is the only available method of connectivity. This case can be found in smart metering, environment monitoring and agricultural applications [2].

LTE uses the multiple access scheme OFDMA with Cyclic Prefix (CP) in the downlink (DL) and Single Carrier Frequency Division Multiple Access (SC-FDMA) with CP in the uplink (UL) [9]. It applies OFDMA because of its robustness to multipath fading effect in wideband channels. It uses Adaptive Modulation and Coding (AMC) with three modulation schemes (QPSK, 16QAM and 64QAM) and different code rates [14].
In LTE, any scheduling algorithm can be executed at each eNodeB by a dynamic packet scheduler (PS) to distribute the available resources to different users, which are under its coverage area, as well as the transmission parameters including the type of modulation and coding scheme (MCS). LTE is designed such that the length of subframe are equal to (1) ms; this value in fact is equal to Transmission Time Interval (TTI). For optimum performance, the resources should be rescheduled every (1) ms, it is worth mentioning that, the assignment of resources could be changed depending upon several factors including CQI (Channel Quality Indicator) which is sent by the user ( $\mathrm{SN}$ ) as a feedback to the eNodeB [10].

Link adaption (LA) is the process of selecting the transmission parameters and MCS. Link Adaption along with the scheduling of resources should be chosen carefully to maximize the cell capacity.

LTE does not define a unique scheduling algorithm. The LTE DL previously is analyzed in several papers like [3], [4] and [5]. Moreover, the user throughput and fairness for a UE (User Equipment) over the simulation time have not been treated. In [7-9], a Kwan Maximum Throughput (KMT) scheduler is proposed that tries to maximize the overall Throughput. In [5], two Proportionally Fair (PF) schedulers that allocate RBs using a localized scheme and an interleaved allocation are proposed. In [8], the Throughput conditions are investigated for two of the most popular scheduling methods, Round Robin and Proportional Fair, to demonstrate a good comparison for downlink transmission.

This paper, investigates the LTE scheduling problems in a monitoring WSN environment. It is organized as follows. Section two, presents an overview of LTE packet scheduling in LTE DL. The simulation results presented and analyzed in Sections 3 and 4. Section 5 concludes the paper.

\section{LTE DOWNLINK RADIO RESOURCE SCHEDULING ALGORITHM}

Scheduling in the downlink LTE system should be performed during one TTI, it consists of two time slots, or resource-block-pair basis (RB, one subframe of $0.5 \mathrm{~ms}$ over $180 \mathrm{kHz}$ ). Within this TTI, two consecutive RBs are assigned to a user $[4,5]$. In each TTI, each SN computes its received signal strength or signal to interference plus noise ratio (SINR) on the reference signals received from the serving eNodeB. Figure 1 shows a generalized model of packet scheduling in the downlink LTE system that consists of $\mathbf{N}$ RBs and $\mathbf{K}$ sensor nodes (SNs). The overall goal of most scheduler is to provide the system and users 
requirement. A good scheduling algorithm therefore has two main objectives: First to maximize the throughput and second to achieve fairness between users. To achieve this goal, there are many algorithms developed for wireless system, such as maximum rate scheduling, round robin (RR), best CQI, proportional fair (PF), max-min and resource fair.

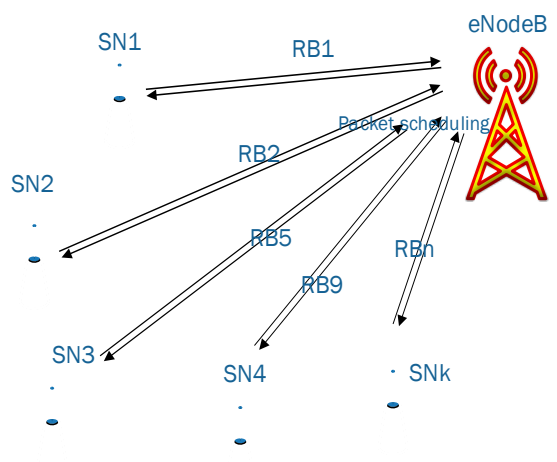

Fig. 1: Packet scheduling operation

\subsection{Round Robin (RR):}

Round Robin (RR) is one of the fundamental and widely used scheduling algorithms. Its running process is very simple and easy to implement. The RR algorithm [12] assigns equal portions of packet transmission time to each user in a circular order. RR algorithm achieves the best fairness performance if the users have similar channel conditions and similar sized packet arriving at their buffers. It is on a first come first served basis. Although RR gives every UE an equal opportunity to obtain RBs, the overall throughput is much lower than in other schedules because this scheduler does not consider the channel conditions. In LTE, different UEs have different services with different QoS requirements and it is very difficult to allow every SN to take up the same RBs for the same possibility because it will decrease the resource efficiency [5].

\subsection{Best CQI Scheduler (BCQI):}

Best CQI scheduling method optimizes the SN throughput by assigning the $\mathrm{RBs}$ to the $\mathrm{SN}$ with the best radio link conditions, meaning that the SNs with low CQI value have the lower chance to be served [11]. This CQI information contains the value of the signal-to-noise and interference ratio (SINR) measured by the SN. A higher value of CQI indicates a better channel condition. The best CQI is selected for scheduling based on the CQI received. BCQI scheduling scheme can increase cell throughput at the expense of worst fairness. In this scheduling mechanism, SNs located far from the base station are unlikely to be schedule.

\subsection{Proportional Fair Scheduler (PF):}

This algorithm assigns the PRBs to the SN with the best channel quality a combination of CQI \& level of fairness desired. There are various versions of PF algorithm based on values it takes into account. Main target of this algorithm is to achieve a balance between Maximizing the cell throughput and fairness, by letting all users achieve a minimum QoS (Quality of Service) [12].

\subsection{Max-Min scheduler}

Maximizing the minimum of the UE throughputs is the main task of Max-Min scheduler. Max-Min scheduler allocates the resources in a way that an equal throughput for all users (SNs) is guaranteed. The scheduler maximizes the minimum of the SN throughputs [14]. Max-Min scheduler is Pareto optimal, meaning that the rate of one SN cannot be increased without decreasing the rate of another $\mathrm{SN}$ that has a lower rate than the one considered.

\subsection{Resource Fair Scheduler (RF):}

The RF scheduler plan allocates an equal amount of resources for all UEs. It mainly aims to maximize the sum rate of all UEs while ensuring fairness with respect to the number of RBs assigned to a UE.

\subsection{Max. Throughput Scheduler (KMT)}

The KMT scheduler performs assignment of resources in two steps in order to reduce complexity. In the first step, each two RBS (i.e. SB) are assigned to the SN who can support the highest bit rate. In the second step, the best MCS for each UE is determined. The idea behind KMT scheduler is to assign a disjoint subset of SBs to each UE, thereby a joint multiuser optimization problem is reduced into $U$ (number of simultaneous users) parallel single-user optimization problems. [14] Provides more details about this schedule.

\section{SIMULATION MODEL ASSUMPTIONS}

We have considered the cellular network with one base station (eNodeB) and 'K' number of sensor nodes SNs, each assumed to have one transmit and one receive antenna. At each time slot, eNodeB assign 'R' RBs to 'K' active users. The proposed system model is shown in figure 2 . A single cell of $10 \mathrm{MHz}$ bandwidth with $50 \mathrm{RBs}$ and $2 \mathrm{GHz}$ carrier frequency is used. The eNodeB has a fixed location at the center of the cell and it controls all the available RBs. These RBs are to be shared by all SNs within the cell. The LTE system level simulator [6, 8] introduced in was used with simulation parameters shown in Table 1.

Due to the simulator's limitations, we do modify the simulator module. Because in order to compare the scheduling algorithm impact on SN throughput, the SNs position with regard to the eNodeB location should be kept when making various simulation scenarios.

Table 1. Simulation Parameters

\begin{tabular}{|c|c|}
\hline Parameter & Value \\
\hline Frequency & $2.0 \mathrm{GHz}$ \\
\hline LTE bandwidth & $10 \mathrm{MHz}$ \\
\hline Number of RBs, N & 50 \\
\hline Number of SNs & varies \\
\hline Number of BS & 1 \\
\hline Inter eNodeB distance & $500 \mathrm{~m}$ \\
\hline Channel Model & $3 \mathrm{GPP}$ TU \\
\hline Simulation length & $1000 \mathrm{TTI}$ \\
\hline SNs position & SNs located in target area. \\
\hline Antenna setup & 1 transmit, 1 receive (1 x 1) \\
\hline
\end{tabular}




\begin{tabular}{|l|ll|}
\hline Receiver & \multicolumn{2}{|c|}{ Zero Forcing ZF } \\
\hline & $\bullet$ & Round Robin (RR) \\
& • & Best CQI (BCQI) \\
& $\bullet$ & Proportional Fair \\
& & (PF) \\
Schedulers & Max. Throughput \\
& & (KMT) \\
& - \\
& Resource Fair \\
& (PF) \\
& & Max-Min \\
\hline
\end{tabular}

\section{SIMULATION RESULTS}

The following results are based on the following features:

1-There are 15 CQI levels in the LTE network and the simulator generates $30 \mathrm{SNs}$ which are distributed in the cell as shown in figure 2 .

2- Selecting SN19, which is located very close to eNodeB to measure the different parameters of the network. Figure 4, shows a high throughput corresponding to the CQI level of 15 (see figure 3 for CQI distribution of node SN19). In other words, level 15 of CQI is used most of the time and is frequently updated by eNodeB 2 . It is possible to conclude that SN19 is in a very good condition to receive the maximum date rate. Such high CQI level will produce low BLER due to the channel conditions as will be shown later.

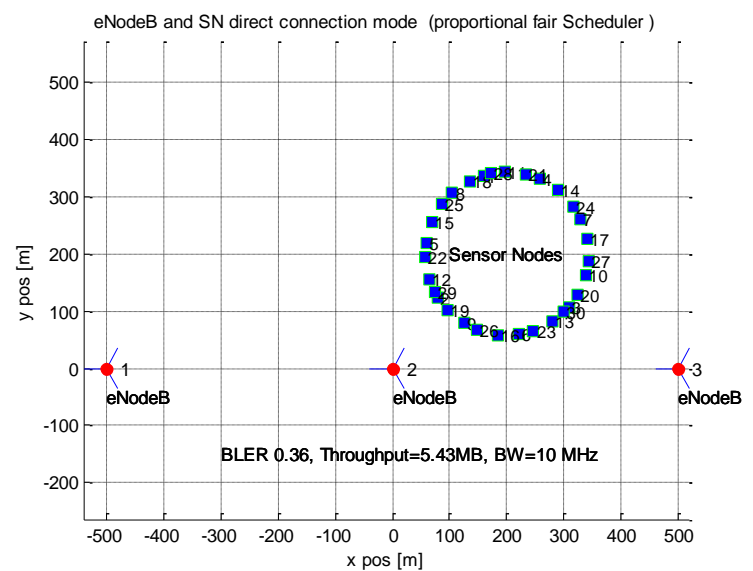

Fig. 2. Shown the relative distances between eNodeB and SNs. Red dot represents eNodeB (eNodeB2 sector 1 is active) and blue dot represents $\mathrm{SN}$.

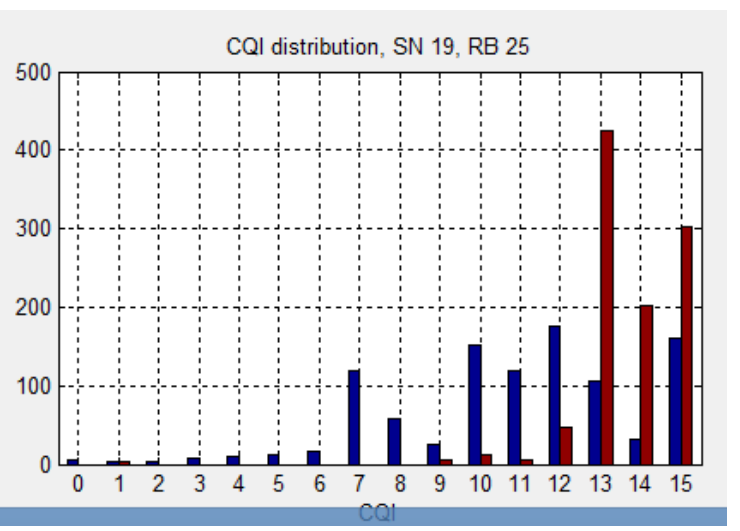

Fig. 3. CQIs distribution of SN19 while is close to eNodeB 2, RB25.
Figures 3 and 4 shows the sent CQI histogram report for the selected resources block RB and stream (blue), mean CQI for the whole frequency band (red) and a CQI of the Transport Block (TB) sent to the $\mathrm{SN}$, if scheduled. The distribution of the CQIs for the selected $\mathrm{SN}$ and RB during the simulation time (blue), and of the TB CQIs (red). Figures 5 and 6 declare the system throughput and BLER as a function of time.

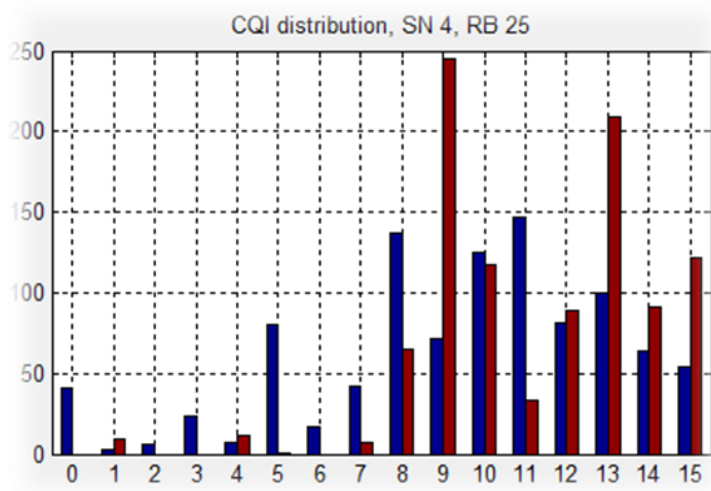

Fig.4. CQIs distribution of SN4 while is far from eNodeB 2, RB25.

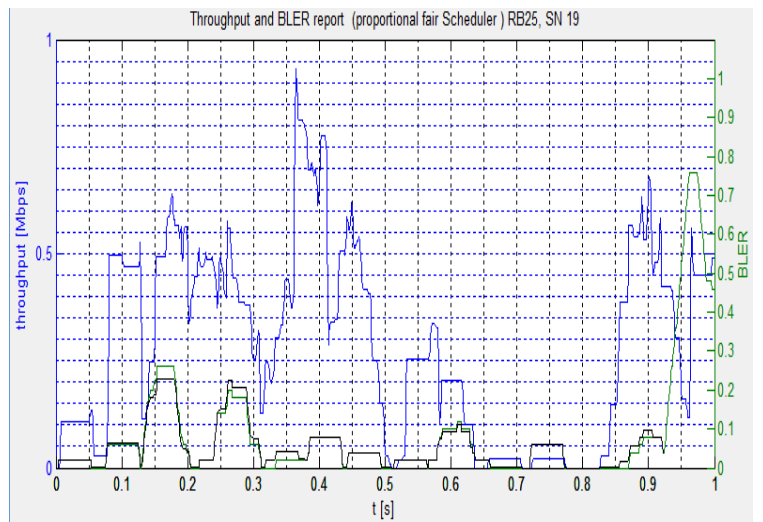

Fig. 5. Throughput, BLER at SN19 while is near eNodeB 2.

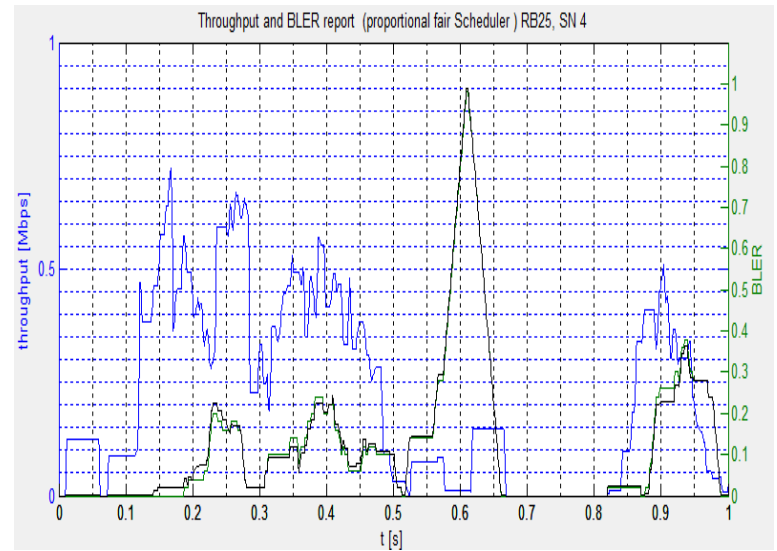

Fig.5. Throughput, BLER at SN4 while is far from eNodeB 2.

To highlight the effect of packet scheduling algorithms on the SN throughput performance, Figure 7, 8 and 9 depict the different SNs throughputs (for actual comparison the SNs keep their places the sure with respect to the eNodeB for all the conducted simulations). As expected, the best CQI 
strategy has the highest SNs throughput. Proportional Fair algorithm stands between RR and Best CQI.

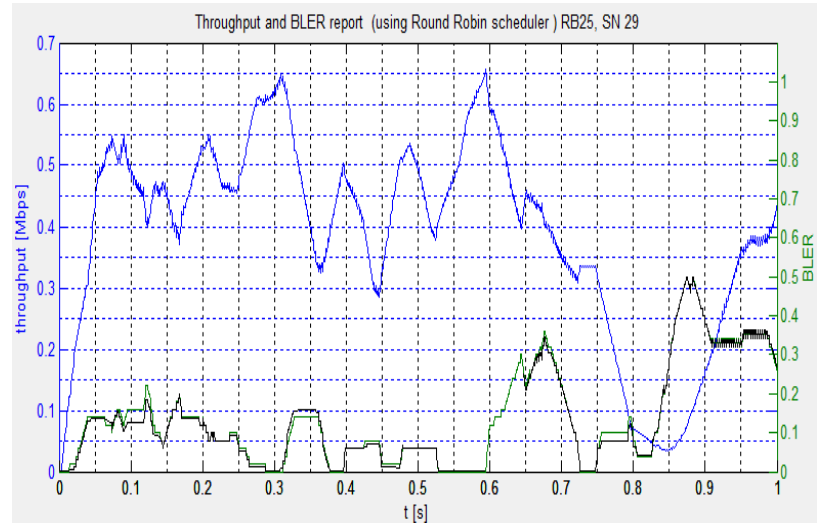

Fig. 7. Throughput and BLER report for SN29 using Round Robin scheduler.

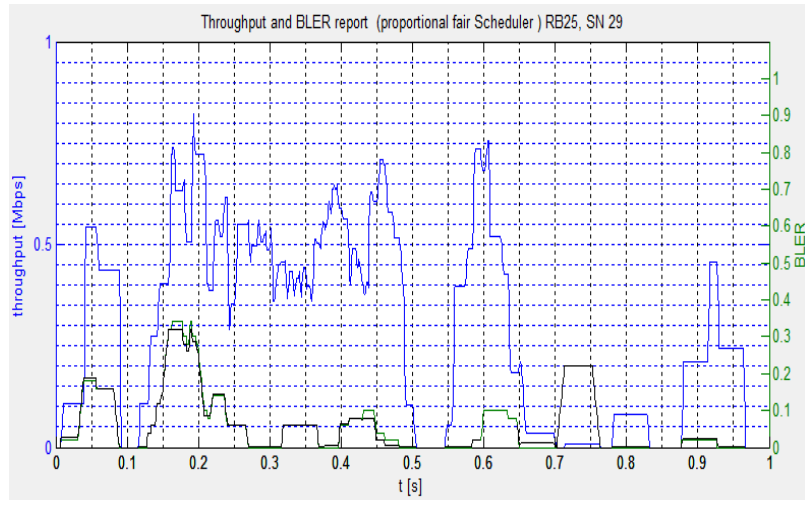

Fig. 8. Throughput and BLER report for SN29 using Proportional Fair Scheduler.

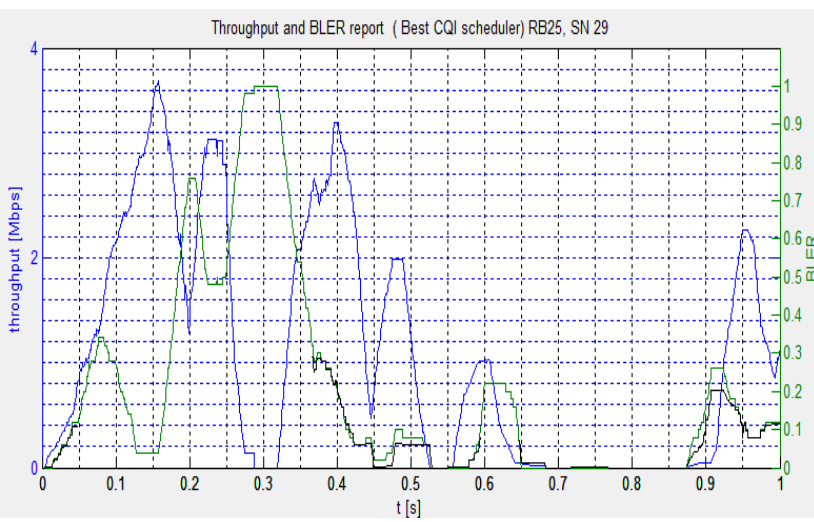

Fig. 9. Throughput and BLER report for SN29 using Best CQI scheduler.

The same simulation scenario for the six scheduling techniques ware used. Figures 10 and 11 shows that, at the first part of the range of SNs, Proportional Fair (PF) performs better than Max-Min. However, with a higher number of SNs, Max-Min outperforms Proportional Fair (PF). At a higher number of SNs, Max. Throughput (MT) outperforms both Proportional Fair and Max-Min schedulers in terms of throughput only while the PF and Max-Min achieve the best tradeoff between fairness and throughput. Figures 10 and 11 shows that the Proportional Fair and Max-Min schedulers outperform the Resource Fair (RF) scheduler in terms of throughput and fairness, thereby resulting in a better tradeoff between fairness and throughput.

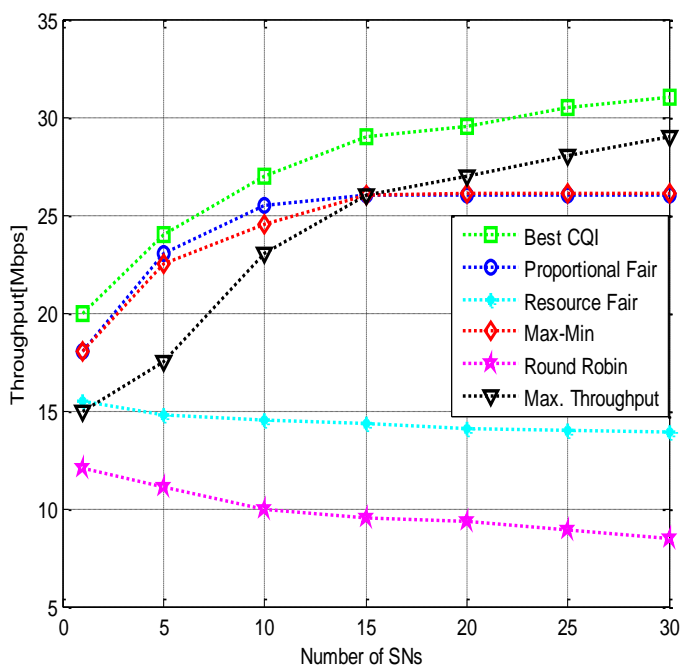

Fig.10 Throughput versus number of SNs with different schedulers.

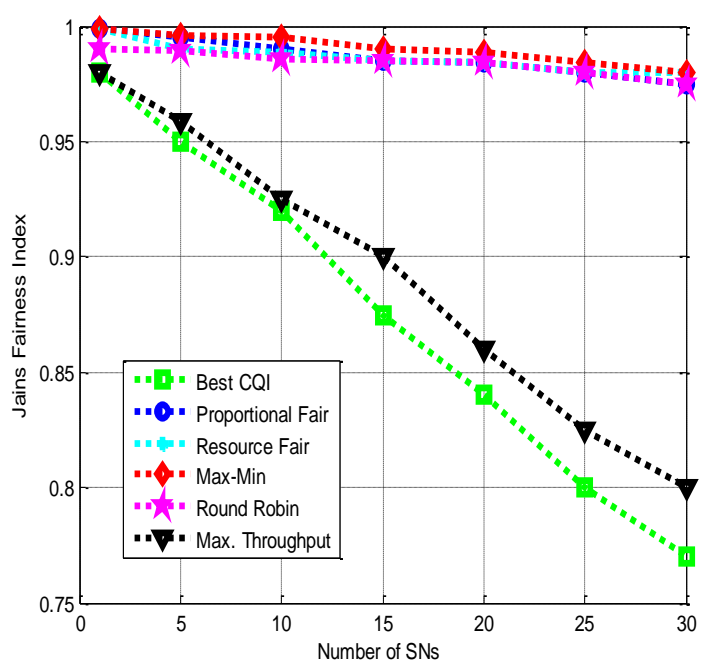

Fig.11 Fairness achieved versus number of SNs with different schedulers.

\section{CONCLUSION}

LTE is one of the best wireless transmission platforms for WSN. In this paper, considered sensor node (SN) directly connect to LTE. Performance for Scheduling in the downlink LTE system was evaluated and compared in terms of throughput, BLER and fairness. Simulation in different scenarios was implemented. The simulation results show that PF and Max-Min schedulers deliver a good compromise between fairness and throughput with low BLER. In addition, we found that the RR scheduler could maintain fairness for all of the connections with lower throughput. Both Best CQI and Max Throughput are sum rate maximizing resource allocation algorithms, which the priority of each $\mathrm{SN}$ assigned is according to the CQI feedback value. The RF and PF schedulers try to maximize the sum rate of all SNs while guaranteeing fairness with respect to the number of RBs an SN gets. Furthermore, the scheduler capability of managing emergency is an interesting prospect for future investigations. 


\section{REFERENCES}

[1] I.F. Akyildiz, W. Su, Y. Sankarasubramaniam, and E. Cayirci, Wireless sensor networks: A survey, Computer Networks (Elsevier) Journal, vol. 38, Issue 4, 15, 2002.

[2] M.W. Beale, S.J. Barrett, P.W. Piggin, P.A. Young; Y. Morioka, APPLICATION OF LTE TO WIRELESS SENSOR SYSTEMS, IP Wireless, 2013.

[3] 3GPPTS 36.300v8.12.0 Release 8, Evolved Universal Terrestrial Radio Access (E-UTRA) and (E-UTRAN), over all description, Stage 2.

[4] O. Yosef and I. Benicia, Performance Analysis of Downlink LTE Using System Level Simulator, U.P.B. Scientific Bulletin, series C, Vol. 75, pp. 111-122, 2013.

[5] Habaebi, et al., Comparison Between Scheduling Techniques in Long Term Evolution, IIUM Engineering Journal\Vol. 14, No. 1, pp. 66-75, 2013.

[6] Mehlfuhrer, et al., The Vienna LTE Simulators Enabling Reproducibility in Wireless Communications Research; EURASIP Journal on Advances in Signal Processing, Vol. 29, July 2011.

[7] J. Ikuno, et al., System Level Simulation of LTE Networks; in Vehicular Technology Conference (VTC 2010-Spring), IEEE 71st, May 2010.

[8] [Online]. Available: http://www.nt.tuwien.ac.at/.

[9] H.A., M.R., R.B., K.S., R.P., Performance of Well Known Packet Scheduling Algorithms in the Downlink 3GPP LTE System, 2009 IEEE ninth Malaysia International Conference on Communications.
[10] F. Capozzi, D. Laselva, F. F., I.Z. Kovacs, P.E.M., UTRAN LTE Downlink System Performance under Realistic Control Channel constraints, (VTC 2009-Fall), pp. 1-5, 2009

[11] O. Iosif, I. Banica, On the analysis of packet scheduling in downlink 3GPP system, CTRQ 2011, Reliability and Quality of Service, pp. 99-102.

[12] M.T.K., A.R.H, H.M. A. B. Farid, Adil M. J. S., Ibrahim K.R., Performance Comparison between Round Robin and Proportional Fair Scheduling Methods for LTE, Department of the Electrical and Electronic Engineering Islamic University of Technology Dhaka, Bangladesh.

[13] Stefania S., Issam T. and Matthew Baker, LTE- the UMTS long-term evolution: from theory to practice (2009 John Wiley \& Sons, Ltd. ISBN: 978-0-470).

[14] SIDDHARTH MAHIDA, ASLAM .S. DURVESH, Design and Performance Evaluation of DL MAC Scheduling Model in LTE, IJRITCC | January 2014 Available @ http://www.ijritcc.org.

[15] 3GPP TSG2RAN Meeting \#53, 3GPPTM Work Item Description: RAN overload control for Machine2Type Communications". Fukuoka (Japan), 2011.

[16] J.T. Entrambasaguas, M.C. Aguayo2Torres, G. Gomez and J.F. Paris, Multiuser capacity and fairness evaluation of channel/QoS2 aware multiplexing algorithms, IEEE Network, pp. 24230, May2June 2007

[17] J. Lim, H. Mung, K. Oh, and D. Goodman, Proportional Fair Scheduling of Uplink Single-Carrier FDMA Systems, (2006) $1-6$. 